\title{
UNA VERDADERA COMUNICACIÓN A PARTIR DEL ARTE: LA RECUPERACIÓN DE LA BELLEZA \\ Y DEL SÍMBOLO, SEGÚN LA CONCEPCIÓN DE MARKO IVAN RUPNIK ${ }^{1}$
}

\author{
MARÍA RUIZ DE LOIZAGA MARTÍN \\ Universidad San Pablo-CEU
}

\begin{abstract}
RESUMEN: Marko Ivan Rupnik subraya que el arte debería hacer percibir lo verdadero y el bien como belleza. La interrelación entre belleza, bien y verdad implica una serie de consecuencias, como la coincidencia de la belleza con la plenitud del ser. Además, la belleza no se puede separar de la comunicación. Esta apertura hacia el otro está relacionada con la recuperación del símbolo. Rupnik destaca que la verdadera obra de arte presenta una concepción simbólica. En ella, la forma está unida al contenido, de modo que la contemplación de la obra puede permitir llegar al conocimiento integral. Los conceptos de belleza y de símbolo que defiende Rupnik están influidos por autores orientales de los siglos XIX y XX. Su estudio nos ayuda a entender los mosaicos que Rupnik realiza como director del Centro Aletti de un modo más completo.
\end{abstract}

PALABRAS CLAVE: Marko Ivan Rupnik; Centro Aletti; belleza; símbolo; arte.

\section{A real communication based on art: the recovery of beauty and sYmbol, According to Marko Ivan Rupnik's Conception}

ABSTRACT: According to Marko Ivan Rupnik, art should reveal the beauty in truth and good. The interrelationships among these concepts have a variety of implications, such as beauty coinciding with the state of fullness of the being. Furthermore, beauty and communication cannot be separated from each other. To the extent communication - and beauty - represents an outward projection towards others, it unveils an undercurrent essence of restoration of symbolism. Rupnik firmly believes that the real work of art must present a symbolic conception. Form is joined to content, thus only if we contemplate the work, can we arrive to the full extent of the knowledge contained in the work. Rupnik's conceptions of beauty and symbol are influenced by $19^{\text {th }}$ and $20^{\text {th }}$ century Eastern Europe authors. Their work helps us understand comprehensively the mosaics created by Rupnik, as the director of the Centro Aletti.

KEY WORDS: Marko Ivan Rupnik; Centro Aletti; beauty; symbol; art

1. RUPNIK: ARTISTA, TEÓlOGO Y SACERDOTE JESUITA EN BÚSQUEDA DE LA BELLEZA

Miembro de la Academia Europea de las Artes y las Ciencias, consultor del Pontificio Consejo para la Promoción de la Nueva Evangelización, y de la Congregación para el Culto Divino y la Disciplina de los Sacramentos, el jesuita Marko Ivan Rupnik (1954, Zadlog, Eslovenia) es el director del Centro Aletti (Roma), cuyas obras musivas se extienden hoy día por América, Oriente Medio, Australia y Europa. Tras completar sus estudios de filosofía, Rupnik se inscribe en la Academia de Bellas Artes de Roma, donde entra en contacto de forma directa con distintas tendencias del arte contemporáneo que han

1 Quisiera expresar mi agradecimiento a la Dra. María Rodríguez Velasco y al Dr. José Francisco Serrano Oceja por sus consejos y todo su apoyo. 
influido fuertemente en sus obras. Continúa sus estudios de teología, se ordena sacerdote y obtiene el doctorado con una investigación dirigida por el cardenal Tomáš Špidlík. Siempre tiene presente que la verdadera vocación jesuita es la disponibilidad a la misión de la Iglesia ${ }^{2}$.

En 1995, Rupnik comienza a dirigir el taller de arte espiritual del Centro Aletti, que forma parte de la Compañía de Jesús. No ha cesado de realizar mosaicos desde que en 1996 san Juan Pablo II le confiara el trabajo artístico en la capilla Redemptoris Mater (Ciudad del Vaticano), hasta la actualidad, propagando una belleza que salva, una visión teológica donde prevalece una aproximación simbólica, litúrgica ${ }^{3}$. De hecho, uno de los principales objetivos de Rupnik es proponer un arte que vivifique la propia liturgia, que haga presente el misterio de la fe, incluso cuando la liturgia no se celebra ${ }^{4}$. Tiene como objetivo crear un espacio donde se provoque una presencia, se revele una persona y se motive el encuentro con Dios ${ }^{5}$. Para ello, busca una armonización entre Oriente y Occidente, así como entre tradición y contemporaneidad en la configuración de estos programas litúrgicos musivos. De esta forma, se perciben reinterpretaciones de tipos iconográficos paleocristianos, bizantinos, románicos, góticos y, al mismo tiempo, influencias de determinados artistas de vanguardia en el lenguaje formal de estos mosaicos, ya que Rupnik es consciente de que sus obras se dirigen al hombre del siglo $\mathrm{XXI}^{6}$.

2. LA BELLEZA, ENCARNACIÓN DEL BIEN Y LA VERDAD: EL CAMINO HACIA LA PLENITUD DEL SER

Rupnik considera que el arte debería hacer percibir lo verdadero y el bien como belleza. Este planteamiento implica que el artista tenga que salir de su punto de vista individual y se preocupe porque se perciba en la creación final

2 Gómez-Oliver, V. y Benítez, J. M., «Entrevista a Rupnik», en: Gómez-Oliver, V. y BeníTEZ, J. M (ed.), 31 jesuitas se confiesan, Barcelona: Ediciones Península, 2003, p. 197.

3 Mattei, G., Il cardinale Tomás Špidlik si racconta alla vigilia del suo compleanno. Da novant'anni con lo sguardo verso oriente, "L'Osservatore Romano", 2009. Recuperado de http://www.vatican.va/news_services/or/or_quo/interviste/2009/290q08a1.html [consultado el 2.03.2018]

4 GoveKar, N., El rojo de la Plaza de Oro. Entrevista de Natasa Govekar con Marko Ivan Rupnik sobre arte, fe y evangelización, Monte Carmelo, Burgos 2013, p. 80.

5 RupnIK, M. I., "Applicazione del Concilio: quale arte per la liturgia?» en: Notitiae, 471472 (2005), p. 179.

6 Son diversas las investigaciones y reflexiones en las que se subraya esta fusión. Podemos destacar: Apa, M., Clément, O. y Valenziano, V. (ed.), La Capilla Redemptoris Mater del Papa Juan Pablo II, Monte Carmelo, Burgos 2002; Rodríguez Velasco, M. (ed.), Tradición y Modernidad en la obra de Marko Iván Rupnik, CEU Ediciones, Madrid 2013; RuIz de LoIzAga Martín, M., «La semplificazione delle forme. Influenza di Matisse nei mosaici di Marko Ivan Rupnik e il Centro Ezio Aletti» en Collectanea Franciscana, 87 (2017), pp. 613-634; o RuIz DE Loizaga Martín, M., «La reinterpretación del descenso de Cristo a los infiernos en los mosaicos litúrgicos del Centro Aletti» en Estudios Eclesiásticos, 94 (2019), pp. 443-475. 
la realidad superior que toda obra contiene ${ }^{7}$. El desvelamiento de esa realidad superior es también apuntado por Gaudí, que señala que «el hombre no crea: descubre y parte de ese descubrimiento ${ }^{8}$, y podría asimismo relacionarse con la idea que desarrolla Romano Guardini: el artista capta la realidad «contemplando su esencia desde su presencia»'.

El director del Centro Aletti se lamenta de que en la actualidad, en muchas ocasiones, el artista no se preocupa por reflejar la belleza en sus obras. De este modo, concluye que "hoy el arte se define sin belleza» y, al considerarse como una realidad separada de la misma, «es realmente complicado decir qué es el arte» ${ }^{10}$. Otros investigadores y artistas también han manifestado la separación, en términos generales, entre la belleza y el arte actual ${ }^{11}$. En concreto, son significativas las palabras de Danto que precisa que los ready-mades fueron apreciados por Duchamp precisamente por no poder ser descritos en términos estéticos, «y él demostró que sí eran arte pero no bellos, la belleza realmente no podría formar parte de ningún atributo definitorio del arte» ${ }^{12}$.

Es importante profundizar en el concepto de belleza que defiende Rupnik, para así entender los mosaicos que realiza el Centro Aletti de un modo más completo, y que se proponen como alternativa a esas otras obras contemporáneas cuyos autores pretenden subrayar la ruptura entre el arte y la belleza. A pesar de que ya en la antigüedad, autores como Platón o Aristóteles apuntaron algunas ideas relativas a la belleza, que los Padres y Doctores de la Iglesia, como san Agustín o santo Tomás, retomarán y vincularán al cristianismo, el concepto de belleza impulsado por Rupnik está principalmente influido por autores orientales de los siglos XIX y XX, por lo que introduciremos en este estudio algunas de sus reflexiones, que corrobora el director del Centro Aletti.

Entre ellos destaca, por un lado, Vladimir Soloviev (1853-1900), filósofo, teólogo, poeta y crítico literario ruso que juega un importante papel en el desarrollo de la filosofía y poesía rusa a finales del siglo XIX. Las ideas principales que sintetizan su pensamiento son: la unidad integral de Dios y del universo; la divino-humanidad de Cristo y de la Iglesia; y la teocracia universal cristiana, es

\footnotetext{
7 RuPNIK, M. I., L'arte memoria della comunione. Il significato teologico missionario dell'arte nella saggistica di Vjačeslav Ivanovič Ivanov, Lipa, Roma 1994, p. 86.

8 Bergòs i Massó, J., Gaudí. El hombre y la obra, Lunwerg, Barcelona y Madrid 2011, p. 34 .

GuARDini, R., Imagen de culto e imagen de devoción. La esencia de la obra de arte, Ediciones Guadarrama, Madrid 1960, p. 45. Véase también: RuIz DE LoIzAga Martín, M., «Hacia un arte litúrgico, según la concepción de Marko Ivan Rupnik. Reflexiones sobre el arte sacro» en: Scripta Theologica 49 (2017), p. 623.

10 RupNIK, M. I., Lectio Academica. La belleza, lugar del conocimiento integral, en: RUPNIK, M. I. (ed.), Una belleza para el encuentro, Universidad Francisco de Vitoria, Madrid 2013, p. 52.

11 Véase: Plazaola, J., Arte sacro actual, BAC, Madrid 2006, p. 13; y Lipovetsky, G. y Serroy, J., La estetización del mundo. Vivir en la época del capitalismo artístico, Anagrama, Barcelona 2015, p. 42.

12 Danto, A. C., Después del fin del arte. El arte contemporáneo y el linde de la historia, Paidós Estética, Barcelona 2010, p. 125.
} 
decir, el reino de Cristo en la tierra. En palabras de Špidlík, Soloviev es «uno de los más auténticos pensadores religiosos rusos» ${ }^{13}$.

Discípulo de Soloviev es Vladislav Ivanovic Ivanov (1866-1949), poeta ruso, autor de numerosos ensayos dedicados al arte ortodoxo y profesor, entre otras instituciones, del Pontificio Istituto Orientale de Roma.

Por su parte, Semen Lyudvigovich Frank (1877-1950), nacido también en Rusia, de donde tuvo que exiliarse en 1922, perteneció a una familia judía, pero en 1912 se convierte al cristianismo. Gran filósofo, es considerado uno de los pensadores que conforman las columnas del llamado renacimiento ruso.

Y, finalmente, Pável Florenski (1882-1937), científico, filósofo, matemático, experto en arte y teólogo, emerge como uno de los pilares de la cultura rusa del siglo XX y una de las grandes figuras del pensamiento universal. Muere fusilado en un Gulag soviético. En palabras de Ivanov, es un hombre de una profunda experiencia espiritual, lo que le permite emprender una serie de investigaciones en las que establece las bases de la iconología ortodoxa ${ }^{14}$.

Rupnik indica que, dentro de la esfera de la creatividad, existe un fundamento subjetivo que es el sentimiento y un principio objetivo que es la belle$\mathrm{za}^{15}$. Precisamente, el fundamento subjetivo es el que lleva a que la belleza y el arte estén ligados a la vida de las personas. La belleza está constituida por una interacción continua con el sujeto que la percibe. De este modo, «más que una cuestión filosófico-estética, el arte es una cuestión vinculada a la vida del hombre», una fuerza creadora de una inteligencia que no puede crear alejándose de la vida, sino solo en la propia vida, solo si tiene en cuenta a las personas ${ }^{16}$. En definitiva, Rupnik impulsa la unión entre el arte y la vida, y así pretende reflejarlo en las obras que realiza en el Centro Aletti.

Por otro lado, como hemos comentado, el arte en general debería hacer percibir lo verdadero y el bien como belleza. De hecho, Rupnik define la belleza - siguiendo principalmente a Soloviev- como «la forma sensible del bien y de la verdad», es decir, su encarnación ${ }^{17}$. Ciertamente, Soloviev subraya la importancia de que el bien, la belleza y la verdad sean una realidad encarnada y no una idea abstracta ${ }^{18}$. Por su parte, Florenski también afirma que la verdad, el bien y la belleza representan un solo principio ${ }^{19}$. La relación entre belleza $\mathrm{y}$ verdad es advertida asimismo por algunos autores del siglo XX, como Von

13 ŠPIDLík, T., Los grandes místicos rusos, Ciudad Nueva, Madrid 1986, p. 339.

14 Ivanov, V., Il grande libro delle icone russe, Edizione Paoline, Milano 1987, p. 196.

15 RupNiK, M. I., L'arte memoria della comunione, o.c., p. 34.

16 Govekar, N., El rojo de la Plaza de Oro, o.c., p. 47. Destaca también en este sentido la concepción de la obra de arte como «huella de una vida», como «vida vivida» (GARAGORRI, P., «La verdad en el arte: la vida vivida» en: Revista de ideas estéticas 96 (1966), p. 313).

17 RupniK, M. I., «A partir de la belleza», en: ŠPIDLIK, T. y RupNIK M. I. (ed.), Teología de la evangelización desde la belleza, BAC, Madrid 2003, p. 520.

18 Solov'Ëv, V., I fondamenti spirituali della vita, Lipa, Roma 1998, p. 22.

19 Florenski, P., La columna y el fundamento de la verdad. Ensayo de teodicea ortodoxa en doce cartas, Sígueme, Salamanca 2010, p. 95. 
Balthasar, y artistas como Gaudí, quien afirma que la «belleza es el resplandor de la verdad; sin la verdad no hay arte» ${ }^{20}$.

La interrelación entre la belleza, el bien y la verdad implica una serie de consecuencias. En primer lugar, si la belleza es la encarnación del bien y de la verdad, se acoge libremente y no se impone. De hecho, si no fuera así, si el bien no se convirtiese en belleza, derivaría, tal y como subraya Rupnik, «en un imperativo tremendo, en un eticismo esterilizante», en una dictadura del fanatismo y del moralismo, que «es capaz de aplastar al hombre e imponer la perfección del individuo como vanagloria, como orgullo» ${ }^{21}$.

Además, si la verdad no fuera comunicada y conocida como belleza, se impondría y se presentaría como una dictadura, lo que da lugar a la ideología ${ }^{22}$. Podríamos decir así, como el director del Centro Aletti defiende, que la verdad es bella por sí misma.

Por otro lado, el bien sin lo verdadero sería una ilusión, y lo verdadero, si no se encarnase en el bien, sería un fanatismo ${ }^{23}$. Rupnik confirma que la dictadura del bien es la suprema expresión del mal ${ }^{24}$. Soloviev también defiende que el bien separado de la verdad y de la belleza es un sentimiento indefinido, la verdad abstracta es una palabra vacía, y la belleza sin el bien y la verdad es un ídolo ${ }^{25}$.

En definitiva, estos efectos revelan la intrínseca relación existente entre estos tres conceptos.

A partir de la identificación de la belleza, el bien y la verdad, se puede extraer otra deducción: como la verdad y el bien se perpetúan, ya que, como explica Rupnik, la verdad es lo que permanece, la belleza es por tanto también eterna $^{26}$. Así, algo que es bello, lo será siempre. El director del Centro Aletti concretamente explica que la belleza no puede tener caducidad, pues las cosas bellas están impregnadas de verdad y de bien, "y lo verdadero y el bien son tales precisamente porque no tienen ocaso ${ }^{27}$. Por tanto, todo arte, cuando es

20 Von Balthasar, H. U., «Arte cristiano y predicación», en: Feiner, J. y LÖHRER, M. (ed.), Mysterium Salutis. Manual de Teología como historia de la salvación, I, Ediciones Cristiandad Madrid 1992, p. 783; y Bergòs I Massó, J., Gaudí. El hombre y la obra, o.c., p. 34.

21 RupNiK, M. I., «Implicaciones teológicas del mosaico», en: Toraño, E. y Prades, J. (ed.), Dios es amor, San Dámaso, Madrid 2009, p. 67.

22 Velasco Quintana, P. y Rodríguez Velasco, M., «Marko Ivan Rupnik. Una nueva forma de creación frente al subjetivismo contemporáneo» en: Debate Actual 13 (2009), p. 120.

23 Rupnik, M. I., A partir de la belleza, o.c., p. 520.

24 RupNIK, M. I., L'autoritratto della Chiesa. Arte, bellezza e spiritualità, EDB Roma 2015, p. 18 .

25 RuPniK, M. I., «Bellezza e pensiero teologico», en: Ravasi, G. y RuPNIK, M. I. (ed.), Il fascino del bello. Tra Bibbia e teologia, San Paolo, Milano 2010, p. 67. Rupnik llega a este pensamiento a partir de las reflexiones de Soloviev. Véase: Solov'ËV, V., «Il significato universale dell'arte», en: Dell'Asta, A. (ed.), Il significato dell'amore e altri scritti, Edizioni R.C. La Casa di Matriona, Milano 1988, pp. 151-154.

26 RUPNIK, M. I., «A partir de la belleza», o.c. p. 520.

27 Govekar, N., El rojo de la Plaza de Oro, o.c., p. 121. En este sentido, Rupnik señala que la belleza al ser eterna, nunca cansa, a diferencia, por ejemplo, de un anuncio publicitario 
verdadero, aspira siempre a la inmortalidad. Y es esta eternidad de la belleza la que imprime su carácter universal. De este modo, ya no solo podemos concluir que algo que es bello lo será siempre, sino que, además puede ser percibido así por todas las personas ${ }^{28}$.

Rupnik plantea que la identificación de la belleza con la verdad y el bien supone también que la belleza coincida con la plenitud del ser, con lo que verdaderamente el ser es ${ }^{29}$. En este sentido, Frank escribe que lo bello encierra el último fundamento del $\operatorname{ser}^{30}$.

De este modo, la belleza no es algo accidental, sino que tiene una connotación intrínseca. Como especifican Guardini y Chenis, no debe ser concebida como una ornamentación superpuesta, sino que es señal de plenitud interior y refleja la perfección de la realidad en conformidad con su esencia ${ }^{31}$. Es por ello por lo que Bernard afirma que «la forma exterior es bella solo porque manifiesta un resplandor interior», y al mismo tiempo especifica que cada ser se inserta en el universo siendo llamado por Dios para encontrar su lugar y para tender hacia la armonía del todo ${ }^{32}$. Precisamente, Frank también había enfatizado esa interrelación entre belleza y armonía en sus escritos, al defender que la belleza coincide con la plenitud del ser y es un signo de su armonía ${ }^{33}$. En definitiva, la concepción de la belleza de Rupnik se encuadra también dentro del ser de las cosas, del conocimiento integral.

Además, la verdad exige el ser, que coincide con el bien. Por tanto, Rupnik, siguiendo a Ivanov, plantea que el primer precepto del artista no tiene que ser expresar la propia voluntad sobre la superficie de las cosas, sino penetrar y anunciar el valor de la esencia. Por otro lado, la belleza tiene una capacidad que transfigura. Concretamente, Rupnik señala que la belleza no es una especie de reflejo de una perfección, sino su encarnación activa, capaz de transfigurar la materia y la historia ${ }^{34}$.

que, «inmediatamente parece bello, pero después produce náuseas» (Velasco Quintana, P. y Rodríguez Velasco, M., «Marko Ivan Rupnik», o.c., p. 120).

28 Cabañas, P., «Aportaciones de la comunión a la creación artística», en: Sancho Fermín, F. (ed.), Estética y espiritualidad. "Via pulchritudinis». La belleza en el arte sagrado, la educación, la música, la arquitectura, el cine, la pintura, Monte Carmelo, Burgos 2012, p. 278.

29 RUPNIK, M. I., «A partir de la belleza», o.c. p. 520; y RUPNIK, M. I., «Bellezza e pensiero teologico», o.c., p. 29.

30 Frank, S. L., L'intangibile. Verso una filosofia della religione, Jaca Book, Milano 1976, p. 239.

31 GuARDINI, R., Imagen de culto e imagen de devoción, o.c., p. 63; y CHENIS, C., «La bellezza-bontá nell'arte sacra» en: Arte Cristiana 816 (2003), p. 207.

32 Bernard, C. A., Teología simbólica, Monte Carmelo, Burgos 2005, pp. 168-169.

33 Frank, S. L., L'intangibile, o.c., p. 245. Esta idea ya había sido expuesta previamente por otros autores, como Dostoievski. Véase: DostoevskiJ, F. M., «La questione dell'arte», en: Gatto, E. (ed.), L'estetica e la poetica in Russia, G. C. Sansoni, Firenze 1947, pp. 295-296.

34 Rupnik, M. I., "Presentazione», en: Farchione, A., Arte e Chiesa. La volontà di un incontro della Chiesa, l'attrazione per lo scontro dell'arte contemporanea, Fede\&Cultura, Verona 2011, p. 7. 
El director del Centro Aletti además defiende que el arte en general suscita la maravilla y, como fascina y atrae, no se impone, sino que crea el «espacio necesario para la libre adhesión ${ }^{35}$. La capacidad del arte de provocar el asombro es también apuntada por otros artistas, como Gaudí, y por investigadores, como Plazaola ${ }^{36}$. En este sentido, Frank escribe que lo bello es aquello que cautiva por su fuerza persuasiva y significado interior ${ }^{37}$. Rupnik retoma estas palabras y añade que esta capacidad de asombro ante lo bello es algo característico del ser humano ${ }^{38}$.

Por otro lado, Cristo ha dicho: «Yo soy el camino, y la verdad, y la vida» (Jn 14,6). Cristo es la revelación sensible de la verdad y la realización del bien. Si el bien y la verdad se identifican con la belleza, esta se identificaría a su vez, por tanto, con Cristo, lo que lleva a Rupnik a deducir que la belleza es necesariamente cristológica. Además, si se plantea que la belleza coincide con la plenitud del ser, se deduce que esta también es cristológica, pues en Cristo se realiza la plena unidad de todo y en Él todo subsiste ${ }^{39}$. Por todo ello, el arte se debe convertir en lugar de encuentro con lo divino y la belleza en aquello que comunica y recuerda a Dios, de tal modo, que emerge, en palabras del director del Centro Aletti, como un mundo penetrado por el Espíritu que revela a Dios, que comunica su vida ${ }^{40}$. Así, la belleza en el arte se podría decir que es un destello de la belleza divina que se manifiesta en la creatividad de los artistas. En este sentido, Maurice Denis ya señaló que la belleza es un atributo de la divinidad ${ }^{41}$.

Si la belleza es Cristo y Él es el amor encarnado, Rupnik plantea que un lenguaje que no está impregnado de amor no será un lenguaje de la belleza ${ }^{42}$. Por tanto, a la interrelación de verdad, belleza y bien, tiene lugar el amor. De esta afirmación también se pueden extraer diversas consecuencias. Por un lado, es lo que le ha llevado a Florenski a concluir que «la verdad manifestada es el amor. El amor realizado es la belleza», y, a partir de estas reflexiones, Rupnik ha insistido en que en una obra de arte que es vivida en el amor se presenta la belleza en el sentido más noble y auténtico ${ }^{43}$. Por otro lado, también podemos

35 Govekar, N., El rojo de la Plaza de Oro, o.c., p. 197.

36 Véase: Gaudí, A., "Conversaciones de Gaudí con Joan Bergós», en: Codinachs, M. (ed.), Antoni Gaudí. Manuscritos, artículos, conversaciones y dibujos, Colegio Oficial de Aparejadores y Arquitectos Técnicos de la región de Murcia, Valencia 2002, p. 91; y PlazaOLA, J., El arte y el hombre de hoy, Servicio de publicaciones de la Diputación Provincial de Valladolid, Valladolid 1978, p. 136.

37 Frank, S. L., L'intangibile, o.c., p. 239.

38 Rupnik, M. I., «L'icona, l'invisibile e la storia» en: Euntes Docete 2 (2000), p. 94.

39 RupniK, M. I., «Bellezza e pensiero teologico», o.c., pp. 68 y 78.

40 RupNIK, M. I., «L'icona, l'invisibile e la storia», o.c., p. 91.

41 Denis, M., Nouvelles Théories sur l'art moderne, sur l'art sacré, 1914-1921, L. Rouart et J. Watelin, Paris 1922, p. 284.

42 Govekar, N. El rojo de la Plaza de Oro, o.c., p. 96-97.

43 Florenski, P., La columna y el fundamento de la verdad, o.c., p. 95; y RUPNIK, M. I., «Presentazione», o.c., p. 7. 
deducir, como hace Rupnik, que donde no hay amor, no hay belleza, y donde no hay belleza, hay mentira, seducción y engaño $0^{44}$.

Asimismo, si la belleza es cristoforme, estaría íntimamente unida a la teología y a la unidad de las disciplinas. Ciertamente, la belleza une y, como dice Soloviev, es siempre una experiencia de unidad, pues cuando la variedad de fenómenos se une en la unidad, entonces percibimos esta armonía visible como belleza ${ }^{45}$. Esta idea también será profundizada por Frank, que plantea que lo bello está compenetrado de una unidad interior, de tal modo que las partes tienen valor y significado no por sí mismas, sino que logran la plenitud en relación con el resto, con su dependencia con las demás ${ }^{46}$. De hecho, como escribe Špidlík, es precisamente a partir de esta visión unitaria como «la belleza salvará al mundo ${ }^{47}$. En este sentido, Rupnik concluye que la belleza exige una visión de cohesión de las ciencias, de los conocimientos y de las culturas ${ }^{48}$. En definitiva, la belleza no puede ser un objeto aislado en el estudio. El director del Centro Aletti además indica que el desmembramiento de la unidad del pensamiento filosófico en la época moderna provoca la aparición de la estética como ciencia autónoma ${ }^{49}$.

Špidlík y Rupnik también explican que el término estética, proviene del griego aísthesis, que significa sentimiento, intuición ${ }^{50}$. Por tanto, la belleza, según esta concepción, se convierte en una realidad de sentimientos. De este modo, el arte no tendría acceso a un conocimiento objetivo, al subrayarse la dimensión del sentimiento. Si, como señaló Baudelaire, el elemento particular de cada belleza proviene de las pasiones, como tenemos pasiones particulares, "tenemos nuestra belleza ${ }^{51}$. La belleza, por tanto, deja de ser universal. Y esta estetización de la belleza, al estar ligada a los sentimientos — cuya característica fundamental es la volatilidad- lleva a un desconcierto, a un arte en el que no se puede distinguir ya la verdad. Por ello, para Rupnik es tan importante la unión entre arte y teología. Reconoce la importancia que ejercen en la realización de los diversos mosaicos del Centro Aletti los Padres de la Iglesia — pues señala que la patrística está unida a la imagen - y otros autores cristianos — tanto de los primeros siglos del cristianismo, como teólogos contemporáneos orientales y occidentales-. De esta forma, la patrística, la teología actual y la tradición occidental y la oriental forman una unidad.

\footnotetext{
44 RUPNIK, M. I., L'arte memoria della comunione, o.c., p. 138.

45 Solov'Ëv, V., I fondamenti spirituali della vita, o.c., p. 22.

46 Frank, S. L., L'intangibile, o.c., pp. 237-238.

47 Špidlík, T., Presentación, en: LóPez SÁEz, F. J. (ed.), La belleza, memoria de la resurrección. Teodicea y antropodicea en Pavel Florenskij, Monte Carmelo, Burgos 2008, p. 11.

48 Rupnik, M. I., «Bellezza e pensiero teologico», o.c. p. 79.

49 RupNIK, M. I., "L'arte sacra e la liturgia», en: Verdon, T. (ed.), Bellezza e vita. La spiritualità nell'arte contemporanea, San Paolo, Milano 2011, p. 119.

50 ŠPIDLík, T. y RUPNIK, M. I., El conocimiento integral. La vía del símbolo, BAC, Madrid 2013, p. XVI.

51 Baudelaire, C., Arte y modernidad, Prometeo Libros, Buenos Aires 2009, p. 24.
} 
Si retomamos la afirmación de que la belleza es cristológica, Rupnik también especifica que el fundamento de la belleza es el rostro de Cristo, «un rostro deshecho, un rostro martirizado, una herida, un costado abierto». De este modo, inicialmente, algo realmente bello puede no atraer tanto como algo que sea formalmente bello. En este sentido, plantea que «la belleza es como el amor, se entiende y se conoce tarde», y por esta razón también señala que «la belleza no agrada inmediatamente porque dentro encierra el drama» ${ }^{52}$.

El director del Centro Aletti especifica que esta belleza que es eterna y que fascina es la belleza pascual. Si, como hemos afirmado, la belleza está impregnada de amor, hemos de tener en cuenta que el amor de Cristo se ha realizado de forma dramática. Por ello, la belleza siempre presenta una nota del drama pascual, pero que se convierte en historia de la salvación. De este modo, «la verdad se hace accesible en la carne que, en el sacrificio del amor, ha pasado por la muerte y sigue viviendo en una cualidad superior, en un cuerpo neumático, glorioso ${ }^{53}$. Lo ha expresado así también Ratzinger quien, en su reflexión, apunta además la íntima relación entre belleza, amor y verdad, que, como hemos visto, es defendida por Rupnik:

Quien es la Belleza misma se ha dejado golpear en el rostro, escupir encima, coronar de espinas - podemos imaginar todo esto de forma emocionante en la Sábana Santa de Turín. Pero es en este Rostro tan desfigurado donde precisamente aparece la auténtica, la extrema belleza: la belleza del amor que llega hasta el fin y que, precisamente en esto, se revela más fuerte que la mentira y que la violencia. Quien ha percibido esta belleza sabe que la verdad, y no la mentira, es la última instancia del mundo [...]. El icono de Cristo crucificado nos libera de este engaño tan extendido hoy. Es más, nos plantea la condición de que nos dejemos herir junto a él y creamos en el Amor, que puede correr el riesgo de abandonar la belleza exterior para anunciar, de este modo, la verdad de la belleza ${ }^{54}$.

Previamente, Frank había escrito que la belleza es un reflejo del paraíso y otros autores señalan que presenta la Parusía ${ }^{55}$. Por todo ello, Rupnik concluye que la esencia de la belleza es la esencia de la escatología ${ }^{56}$. Entonces retoma la conocida frase de Dostoievski, para recordar que la belleza ya ha salvado y está salvando el mundo ${ }^{57}$.

52 RupNIK, M. I., «A partir de la belleza», o.c. p. 530.

53 RupNIK, M. I., «Lectio Academica. La belleza, lugar del conocimiento integral», o.c., p. 22 .

Ratzinger, J., La belleza. La Iglesia, Ediciones Encuentro, Madrid 2006, p. 21.

55 Frank, S. L., L'intangibile, o.c., p. 245; y Clément, O., «Un Occidente fecundado por Oriente», en: ApA, M., Clément, O. y Valenziano, V. (ed.), La Capilla Redemptoris Mater del Papa Juan Pablo II, Monte Carmelo, Burgos 2002, p. 185.

56 RupNIK, M. I., «L'artista dell'arte per il culto» en: Arte Cristiana 797 (2000), p. 108.

57 RupNIK, M. I., Vassilij Kandinkij come approccio a una lettura del significato teologico dell'arte moderna alla luce della teología russa, Licenza in missiologia della Pontificia Università Gregoriana. Direttore: Heinrich Pfeiffer, Roma 1987, p. 37. 
En este sentido, resulta revelador que Rupnik hable de una belleza para el encuentro $^{58}$, pues manifiesta la necesaria alteridad de la verdadera belleza y su apertura al Otro con mayúsculas, lo que remite directamente a la belleza cristológica y, por tanto, a la revelación de la plenitud del ser y a la indisoluble unidad entre dicha belleza, el bien y la verdad. Precisamente, este es el objetivo que persigue el Centro Aletti con la realización de sus mosaicos: hacer que se transfigure el espacio litúrgico en el que habitan sus obras, de tal modo que se estimule el encuentro entre el fiel y Dios.

\section{El SÍMBOLO Y SU CORRESPONDENCIA CON EL CONOCIMIENTO INTEGRAL}

Como explican Špidlík y Rupnik, «vivimos en una sociedad que podría llamarse "visiva", llena de imágenes» y, sin embargo, «la gente no está educada para "leer" su significado y ni siquiera los artistas se esfuerzan por dar a las pinturas un profundo significado espiritual $»^{59}$. Esta situación ha sido percibida por otros autores, como Gombrich, quien escribe que nuestra época es visual, por lo que es importante aclarar las posibilidades de la imagen en la comunicación ${ }^{60}$.

De hecho, Rupnik afirma que la belleza no se puede separar de la comunicación y, junto con Špidlík, plantea que la profunda destrucción de una imagen artística y su pérdida de sentido se produce cuando los espectadores se muestran incapaces de comprender lo que el artista ha querido expresar con ella ${ }^{61}$. De esta forma, en esta sociedad visiva, en esta cultura de la imagen, se hace necesario que el código utilizado por el artista sea descifrable; en definitiva, «nace la exigencia de un lenguaje trans-individual que se abra a la alteridad y dé razón de ser en su complejidad ${ }^{62}$.

Por otro lado, recordemos también que el arte está íntimamente ligado a la belleza, que a su vez se identifica con el bien, la verdad, y se relaciona íntimamente con el amor. Es el amor el que permite salir de uno mismo e incluir al otro. Así, la esperanza de que el arte pueda realmente comunicar hoy reside en la inclusión del otro. Esta apertura está relacionada con la recuperación del símbolo, pues como explica Špidlík, la manifestación simbólica supone que el conocimiento sea dialogal, que nazca en la comunicación viva entre las personas $^{63}$.

58 RuPNIK, M. I., Una belleza para el encuentro, Universidad Francisco de Vitoria, Madrid 2013.

59 ŠPidLík T. y RuPNiK, M. I., El conocimiento integral, o.c., p. 58.

60 Gombrich, E. H., La imagen y el ojo. Nuevos estudios sobre la psicología de la representación pictórica, Alianza Forma, Madrid 1991, p. 130.

61 ŠPIDLík, T. y RUPNIK, M. I., El conocimiento integral, o.c., p. 113.

62 Špidlík, T. y Rupnik, M. I., La fe según los iconos, Monte Carmelo, Burgos 2003, p. 9.

63 Špidlík, T., Alle fonti dell'Europa. In principio era l'arte, Lipa, Roma 2006, p. 76. 
Además, Rupnik ha subrayado que hoy estamos en una época orgánica. Para entender esta afirmación es necesario definir la época orgánica, y su diferenciación con lo que el director del Centro Aletti llama época crítica. La cultura crítica - como la desarrollada en la Grecia clásica o en el Renacimiento- se caracteriza por el primado de la idea y del pensamiento, lo que dificulta la presencia del símbolo ${ }^{64}$. El arte que se crea en este periodo tiende a la perfección de la forma, por lo que es realmente una idealización, una realización según las ideas $^{65}$. En cambio, en la cultura orgánica — como la egipcia o la paleocristiana-destaca la mentalidad simbólica y la comunión emerge como su característica principal. Esta sucesión de las diferentes culturas es también explicitada por Ivanov o Florenski ${ }^{66}$.

Como hemos comentado, Rupnik afirma que hoy nos encontramos en una época orgánica. Concretamente, plantea que el inicio del siglo XX es ya el comienzo de la misma, pues cree que al final de un periodo en el que el arte se ha convertido en el ámbito de protesta del sujeto, dominado por formalismos y subjetivismos, parece que de nuevo puede llegar a descubrir su misión de servicio al bien y a la verdad, y manifestarse como belleza. Y concluye que ciertamente hoy el hombre advierte la necesidad de contemplar algo y exclamar «iqué bello!» ${ }^{67}$. De nuevo, Rupnik ha llegado a esta conclusión a partir del pensamiento desarrollado por otros autores, como Berdiaev, que escribe que el mundo se inclina hacia un orden espiritual análogo al de la Edad Media, caracterizado por el fin del individualismo, del liberalismo formalista y el comienzo de una época de nueva colectividad religiosa ${ }^{68}$.

En definitiva, Rupnik señala que hoy nos encontramos en el momento apropiado para que se desarrolle un arte que no busque la perfección formal y, de ese modo, pueda subrayarse su capacidad simbólica, que está íntimamente

${ }^{64}$ Universidad Pontificia Comillas (3.11.2015), Lección Magistral de Teología. Marko RUPNIK. Discernimiento de la cultura a través del arte [Archivo de video]. Recuperado de:

https://tv.comillas.edu/media/Lecci\%C3\%B3n+Magistral+de+Teolog\%C3\%ADa\%2C+Mar ko+Rupnik.+ +3+11+2015/1_mxf6bwh0/66601841 [Consultado el 3.12.2017]

65 RupNIK, M. I., «L'arte formatrice del cuore sacerdotale» en: Seminarium, A.L., 1 (2010), p. 161.

${ }^{6}$ Ivanov, V. I., «Il simbolismo e la grande arte», en: Lo Gatto, E. (ed.), L'estetica e la poetica in Russia, G. C. Sansoni-Editore, Firenze 1947, pp. 478-481; FlorensKis, P., Lo spazio e il tempo nell'arte, Adelphi Edizioni, Milano 1995, pp. 133-134. Burckhardt y Von Balthasar desarrollan planteamientos similares. Véase: Burckhardt, T., Principios y métodos del arte sagrado, Olañeta, Barcelona 2000, p. 81; y Von BaLthaSAR, H. U., Arte cristiano y predicación, o.c., p. 786.

67 Rupnik, M. I., «Verso un'arte sapienziale», en: Guzzi, M. (ed.), Lo spartiacque. Ciò che nasce e ciò che muore a Occidente, Paoline, Milano 2006, p. 172.

68 Berdiaeff, N., Una nueva Edad Media. Reflexiones acerca de los destinos de Rusia y de Europa, Editorial Apolo, Barcelona 1951, pp. 91 y 104; y BERDIAEv, N., El sentido de la historia. Experiencia de la filosofía del destino humano, Ediciones Encuentro, Madrid 1979, p. 158. Asimismo, podemos destacar las reflexiones de: DostoevsкiJ, F. M., «La questione dell'arte», o.c., p. 298; y de Florenskis, P. A., La concezione cristiana del mondo, Edizioni Pendragon, Bologna 2011, pp. 50-53. 
ligada a la época orgánica ${ }^{69}$. Pero, ¿qué es el símbolo? ¿Cuál es esa capacidad simbólica de la que Rupnik habla y que busca mostrar en las obras que realiza como director del Centro Aletti?

En primer lugar, es necesario hacer algunas precisiones, pues el concepto de símbolo puede resultar equívoco. Como el propio Rupnik advierte, hay que percatarse de los planteamientos del pensamiento moderno que, en muchas ocasiones, relacionan el símbolo con una entidad abstracta, o con signos entre los que se instaura una relación definida por las convenciones culturales de un código comunicativo concreto $^{70}$.

Rupnik, siguiendo a Ivanov y Berdiaev, defiende un simbolismo realista ${ }^{71}$. En el simbolismo realista, el símbolo es el puente que pone en comunión al artista con la realidad que se hace presente por medio de él. El símbolo invita a descubrir en una realidad, otra más profunda, por lo que obliga a tener en cuenta una realidad que es más fuerte que aquello que piensas o querrías. Es importante puntualizar que los símbolos no se inventan, sino que se descubren $^{72}$.

El verbo griego symbállein significa poner juntamente, reunir. De hecho, el símbolo hace participar al artista en la unidad de dos mundos: el visible y el invisible. El mundo visible está permeado y compenetrado por el mundo invisi$b^{73}$. El símbolo es así una cierta unidad de lo trascendente y de lo inmanente, un puente entre el cielo y la tierra, una unidad divino-humana ${ }^{74}$.

Por otro lado, recordemos que la concepción de belleza que defiende Rupnik está ligada al ser de las cosas, al conocimiento integral. Esta reflexión está íntimamente relacionada con el concepto de símbolo, pues precisamente este pertenece a un conocimiento integral, como también subraya el director del Centro Aletti. El arte no solo expresa sentimientos y opiniones del artista, sino

69 RuPNIK, M. I., «L'arte sacra e la liturgia», o.c., p. 146.

70 RupNIK, M. I., «Le chiese, icone della Chiesa» en: Thema. Rivista dei Beni Culturali Ecclesiastici 5 (2015), p. 17. Florenski y Evdokimov se percatan también del uso trivial que, en ocasiones, se hace de ciertas palabras, como el símbolo. Véase: FloRENSKIJ, P.A., Il simbolo e la forma. Scritti di filosofia della scienza, Bollati Boringhieri, Torino 2012, p. 189; y EvDoкıмov, P., El amor loco de Dios, Narcea, Madrid 1990, 25.

71 Berdiaev, N., Spirito e libertà, Edizioni di comunità, Milano 1947, p. 99. Véase también: RuPNIK, M. I. L'arte memoria della comunione, o.c., p. 81.

${ }_{72}$ RupNIK, M. I., «Il simbolo: la proposta dei cristiani per l'arte», en: Ricci, F. C. (ed), Il cristianesimo fonte perenne di ispirazione per le arti, Edizioni Scientifiche italiane, Napoli 2004, pp. 155-157.

${ }_{73}$ RuPNIK, M. I., «Per una teologia dell'arte ossia la teologia come creazione artistica», en: Š́IDlík, T. y RuPNIK, M. I. (ed.), Parola e immagine, Lipa, Roma 1995, p. 92.

74 ŠPIDLík, T. y RupNIK, M. I., El conocimiento integral, o.c., p. 171. Berdiaev plantea que el símbolo es el puente que une dos mundos, de forma que el sentido de uno reside en el otro (BerdiaEv, N., Spirito e libertà, o.c., p. 95). Evdokimov retoma el origen etimológico de la palabra para hacer ver que el símbolo implica la unión de dos mitades: el simbolizante y lo simbolizado (Evdokimov, P., El amor loco de Dios, o.c., p. 25). Y Bernard subraya que el símbolo quiere desvelar el profundo significado de la vida humana (BERnARD, C. A., Teología simbólica, o.c., p. 62). 
que es la vía para llegar al conocimiento de los misterios de la verdad misma ${ }^{75}$, tal y como explicitan Špidlík y Rupnik en el título de su investigación: El conocimiento integral. La vía del símbolo.

Rupnik defiende que a través del arte se promueve una visión contemplativa de la unidad, se comunica una visión de conjunto ${ }^{76}$. Esta concepción del conocimiento integral ha sido desarrollada por el director del Centro Aletti de nuevo a partir de su lectura de investigaciones realizadas por diversos autores orientales. Entre ellos, cabe destacar en primer lugar a Soloviev, quien escribe que es necesario que todos los elementos particulares se encuentren interrelacionados en el conjunto, de tal forma que cada uno sienta en su particularidad la unidad con lo global ${ }^{77}$.

Junto a Soloviev, el propio Rupnik señala que debemos referirnos a las enseñanzas de Florenski e Ivanov, pues ambos conciben la creación artística como una especie de intuición que impulsa al artista a dejarse atraer por el Misterio $^{78}$. Concretamente, Ivanov indica que el valor simbólico del arte reside en su concordancia con las leyes de la realidad superior ${ }^{79}$. Además, desarrolla y explica el movimiento que se produce en el momento de la creación artística. Así, primeramente, el artista debe ascender a la verdad plena. Esa realidad superior es la que crea el vínculo comunicativo. Posteriormente, tras haber ascendido, el artista debe descender y encontrar la forma para dotar al contenido de un lenguaje que deje transparentar la verdad interna. En palabras de Rupnik, el artista debe encontrar la forma que ayude a las personas a reconocer con asombro una realidad superior ${ }^{80}$. Es lo que Ivanov llama la relación entre las realia —el aspecto externo de las cosas_- y las realiora - la parte más interna, el valor ontológico del ser ${ }^{81}$ - Como subraya Ivanov, se puede decir que muchos son los que ascienden, pero pocos los que saben descender. Y precisamente son estos últimos los verdaderos artistas ${ }^{82}$.

Finalmente, Florenski, como escribe López Sáez, señala que la analogía más cercana de la actividad integradora de la razón podría ser la actividad artísti$\mathrm{ca}^{83}$. Además, este autor ruso también subraya la necesaria comunión que debe existir entre las distintas partes de una obra. Asimismo, distingue las diversas

75 Rupnik, M. I., «Cómo me he acercado al mosaico de la Capilla», en: ApA, M., Clément, O. y Valenziano, V. (ed.), La Capilla Redemptoris Mater del Papa Juan Pablo II, Monte Carmelo, Burgos 2002, p. 183.

76 RUPNIK, M. I., Vassilij Kandinkij come approccio a una lettura del significato teologico dell'arte moderna alla luce della teología russa, o.c. p. 42.

77 Solov'ËV, V., «Il significato universale dell'arte», o.c, p. 153.

78 RUPNIK, M. I., «Presentazione», o.c, p. 6.

79 Ivanov, V. I., «I limiti dell'arte», en: Lo Gatto, E. (ed.), L'estetica e la poetica in Russia, G. C. Sansoni-Editore, Firenze 1947, p. 466.

80 RupNIK, M. I., L'arte memoria della comunione, o.c., p. 87.

81 Véase: Ivanov, V. I., «I limiti dell'arte», o.c, p. 459.

82 Ivanov, V. I., «I limiti dell'arte», o.c., p. 452.

83 LÓPEz SÁEz, F. J., La belleza, memoria de la resurrección. Teodicea y antropodicea en Pavel Florenskij, Monte Carmelo, Burgos 2008, pp. 198-200. 
fases de la creación artística -más específicamente, él se centra en el icono-. En estas fases también encontramos movimientos ascendentes y descendentes, en un camino marcado por la ascesis ${ }^{84}$.

Este conocimiento integral y, por tanto, esta unidad que se persigue en el arte, se refleja en la unión de la forma y contenido de la obra artística. Como subraya el director del Centro Aletti, el lenguaje utilizado por el artista no es indiferente, sino que, de algún modo, participa del contenido. Así también el lenguaje es ya en sí mismo una cierta comunicación ${ }^{85}$.

Por tanto, según Rupnik, la verdadera obra de arte presenta una concepción simbólica, y en ella la forma está unida al contenido, de tal modo que la contemplación de la obra puede permitir llegar al ser de las cosas. No obstante, es interesante precisar que, a pesar de que el símbolo se comunique inicialmente de modo inmediato y experiencial, es necesaria una codificación, ya que al unir en su estructura diversos niveles de la existencia, puede ser comprendido con varios significados, pero todos en cualquier caso orgánicamente ligados ${ }^{86}$.

Precisamente, estos múltiples estratos que una obra de arte puede contener debido a su capacidad simbólica son los que permiten la verdadera comunicación. El espectador así no se queda tan solo en la superficie, sino que la obra de arte le deja entrever los estratos más profundos. De este modo, el arte es más comunicativo cuando es simbólico.

Por otro lado, como se ha señalado en la reflexión sobre el concepto de belleza, el arte está vinculado a la vida de las personas. Además, Rupnik traza un paralelismo entre el arte actual y el sacramento de la confesión. De hecho, defiende que esta cierta dimensión de confesión está ligada al arte en general, pues "para ser un verdadero artista, no basta con mirar desde lejos lo que quieres expresar. Debes tener experiencia de ello» ${ }^{87}$. Por tanto, la confesión del artista al realizar la obra de arte enfatiza su relación con la vida. Asimismo, el planteamiento de que el arte verdadero es el simbólico lleva a Rupnik a indicar la unidad entre el arte y la vida, pues el símbolo y la realidad están estrechamente unidos ${ }^{88}$. La íntima unión entre arte y vida también fue impulsada por Florenski, y la estrecha relación entre símbolo y realidad es defendida por otros autores, como Schmemann y Muzj ${ }^{89}$.

84 FlorenskiJ, P. A., Bellezza e liturgia. Scritti su cristianesimo e cultura, Arnoldo Mondadori Editore S.p.A, Milano 2010, pp. 31-32.

${ }_{85}$ RUPNIK, M. I., «Per una teologia dell'arte ossia la teologia come creazione artistica», o.c., p. 85.

86 RUPNIK, M. I., «L'arte sacra e la liturgia», o.c, p. 174.

87 Govekar, N., El rojo de la Plaza de Oro, o.c., p. 53.

88 RUPNIK, M. I., «L'icona, l'invisibile e la storia», o.c., p. 92.

89 Florenskis, P., Lo spazio e il tempo nell'arte, o.c., p. 15; Schmemann, A., Chiesa, mondo e missione, Roma: Lipa 2014, p. 43; y MuzJ, M. G., «Vita nello spirito e simbolo: una relazione necessaria nel pensiero di Ch. A. Bernard», en: MuzJ, M. G. (ed.), Simbolo cristiano e linguaggio umano. Per una piena reintegrazione della teologia simbolica nella teologia, Vita e pensiero, Milano 2013, p. 155. 
El símbolo, además, lleva a que la relación entre el cognoscente y lo conocido esté vivificada por el amor, lo que permite que se trate de un vínculo libre. De este modo, el símbolo crea el espacio de libre adhesión ${ }^{90}$. Como explica entonces Rupnik, si unos padres se santifican amando, un artista se santificará del mismo modo y así manifestará el arte de la vida ${ }^{91}$.

\section{CONCLUSIONES}

En definitiva, Rupnik plantea que el arte está íntimamente ligado a la vida de las personas, y, por tanto, «tiene una extraordinaria fuerza comunicativa y es capaz de sintetizar todos los demás lenguajes del hombre, precisamente porque lo involucra más íntegramente, lo cual se traduce en una comunicación más inmediata». Es por ello por lo que defiende la necesidad de redescubrir en la actualidad un lenguaje artístico que esté íntimamente ligado a la vida ${ }^{92}$. Y, para ello, propone recuperar el concepto de símbolo propio del arte cristiano.

Como también escribe Rupnik, la mentalidad simbólica que el cristianismo ha elaborado y aportado al mundo es una de las grandes novedades que introduce. Los cristianos descubren una solución a la relación entre el mundo divino y humano, a partir del encuentro con Cristo. De este modo, afirman una mentalidad simbólica, es decir, una mentalidad capaz de pensar de forma conjunta dos mundos y en la que, al mismo tiempo, haya una unidad orgánica y libre ${ }^{93}$. En definitiva, se trata de un símbolo como manifestación, revelación y comunicación del Misterio como vida. Asimismo, se crea un espacio para comunicarse con Dios y con el hombre y se fomenta un tejido de encuentros, pues Rupnik defiende que la comunicación se produce a través de un encuentro, ya que lo que se comunica no es una cosa, sino una Persona ${ }^{94}$. De este modo, el arte cristiano no se preocupa por una perfección formal, no porque sus artífices no sean capaces de conseguirla, sino porque defienden otro planteamiento: el que a través de sus obras se pueda ver la realidad como es en Cristo. Este hecho además permite tener una visión unitaria del mundo ${ }^{95}$.

Como hemos señalado, el símbolo une en su estructura diversos niveles de la existencia, por lo que para comprender el símbolo que introduce el cristianismo en su totalidad, es necesaria una iniciación a la memoria y a la tradición de la Iglesia, lo que permite, además, salir del subjetivismo y tener en cuenta al otro ${ }^{96}$.

\footnotetext{
90 Rupnik, M. I., «Cómo me he acercado al mosaico de la Capilla», o.c., p. 183.

91 Rupnik, M. I., L'autoritratto della Chiesa, o.c., p. 23.

92 GoveKar, N., El rojo de la Plaza de Oro, o.c., p. 48 y 79.

93 RUPNIK, M. I., "L'arte sacra e la liturgia», o.c., p. 133.

94 RUPNIK, M. I., «L'arte formatrice del cuore sacerdotale», o.c., p. 165.

95 GoveKar, N., El rojo de la Plaza de Oro, o.c., p. 75 y 152. Véase también: Berdiaev, N., El sentido de la historia, o.c., p. 123.

96 RupNIK, M. I., «L'arte sacra e la liturgia», o.c., pp. 147-148.
} 
Por tanto, la recuperación del lenguaje simbólico puede fomentar el encuentro del arte y la teología. Precisamente, Etsuro Sotoo, que desde 1978 trabaja como escultor en la construcción del Templo Expiatorio de la Sagrada Familia de Barcelona, confiesa que su conversión al cristianismo se produjo a partir del desvelamiento del simbolismo presente en este templo ${ }^{97}$. El Centro Aletti persigue con sus obras lograr esa recuperación de la capacidad simbólica del arte, para que sus mosaicos promuevan la comunicación, revelen el misterio $\mathrm{y}$ fomenten tejidos de encuentros. En definitiva, se busca fomentar un estudio del arte paleocristiano, del primer período bizantino, románico, del primer gótico para ofrecer así al artista contemporáneo «la posibilidad de llegar a tener la misma mirada del iconógrafo y del escultor de aquellas épocas, y al mismo tiempo de hablar con el lenguaje de hoy. Es decir, de hacer la síntesis de estas dos realidades», concluye Rupnik ${ }^{98}$.

\section{BiBLIOGRAFÍA}

Apa, M., Clément, O., y Valenziano, V. (ed.) (2002). La Capilla Redemptoris Mater del Papa Juan Pablo II. Burgos: Monte Carmelo.

Baudelaire, C. (2009). Arte y modernidad. Buenos Aires: Prometeo Libros.

Berdiaeff, N. (1951). Una nueva Edad Media. Reflexiones acerca de los destinos de Rusia $y$ de Europa. Barcelona: Editorial Apolo.

Berdiaev, N. (1947). Spirito e libertà. Milano: Edizioni di comunità.

Berdiaev, N. (1979). El sentido de la historia. Experiencia de la filosofía del destino humano. Madrid: Ediciones Encuentro.

Bergòs i Massó, J. (2011). Gaudí. El hombre y la obra. Barcelona y Madrid: Lunwerg.

Bernard, C. A. (2005). Teología simbólica. Burgos: Monte Carmelo.

Burckhardt, T. (2000). Principios y métodos del arte sagrado. Barcelona: Olañeta.

Cabañas, P. (2012). «Aportaciones de la comunión a la creación artística», en: Sancho Fermín, F. (ed.), Estética y espiritualidad. «Via pulchritudinis». La belleza en el arte sagrado, la educación, la música, la arquitectura, el cine, la pintura. Burgos: Monte Carmelo, pp. 269-289.

Chenis, C. (2003). «La bellezza-bontá nell'arte sacra» en: Arte Cristiana 816, pp. 205-212.

Clément, O. (2002). «Un Occidente fecundado por Oriente», en: Apa, M., Clément, O. y Valenziano, V. (ed.), La Capilla Redemptoris Mater del Papa Juan Pablo II. Burgos: Monte Carmelo, pp. 185-194.

Danto, A.C. (2010). Después del fin del arte. El arte contemporáneo y el linde de la historia. Barcelona: Paidós Estética.

Denis, M. (1922). Nouvelles Théories sur l'art moderne, sur l'art sacré, 1914-1921. Paris: L. Rouart et J. Watelin.

Dostoevskij, F. M. (1947). «La questione dell'arte», en: Gatto, E. (ed.), L'estetica e la poetica in Russia. Firenze: G. C. Sansoni, pp. 290-307.

Evdokimov, P. (1990). El amor loco de Dios. Madrid: Narcea.

97 Sotoo, E. y Almuzara, J. M., De la piedra al Maestro, Madrid: Palabra, 2010, p. 89.

98 Mattei, P., «Un arte que despierta veneración», 30DIAS, (2008). Recuperado de http:// www.30giorni.it/articoli_id_17853_12.htm [consultado el 1.07.2018]. 
Florenskij, P. (1995). Lo spazio e il tempo nell'arte. Milano: Adelphi Edizioni.

Florenskij, P. A. (2010). Bellezza e liturgia. Scritti su cristianesimo e cultura. Milano: Arnoldo Mondadori Editore S.p.A.

Florenski, P. (2010). La columna y el fundamento de la verdad. Ensayo de teodicea ortodoxa en doce cartas. Salamanca: Sígueme.

Florenskij, P. A. (2011). La concezione cristiana del mondo. Bologna:Edizioni Pendragon.

Florenskij, P. A.(2012). Il simbolo e la forma. Scritti di filosofia della scienza. Torino: Bollati Boringhieri.

Frank, S. L. (1976). L'intangibile. Verso una filosofia della religione. Milano: Jaca Book.

Garagorri, P. (1966). «La verdad en el arte: la vida vivida» en: Revista de ideas estéticas 96, pp. 309-313.

Gaudí, A. (2002). «Conversaciones de Gaudí con Joan Bergós», en: Codinachs, M. (ed.), Antoni Gaudí. Manuscritos, artículos, conversaciones y dibujos, Colegio Oficial de Aparejadores y Arquitectos Técnicos de la región de Murcia, Valencia, p. 91.

Gombrich, E. H. (1991). La imagen y el ojo. Nuevos estudios sobre la psicología de la representación pictórica. Madrid: Alianza Forma.

Gómez-Oliver, V. y Benítez, J. M. (2003). «Entrevista a Rupnik», en: Gómez-Oliver, V. y Benítez, J. M (ed.), 31 jesuitas se confiesan. Barcelona: Ediciones Península, pp. 183-199.

Govekar, N. (2013). El rojo de la Plaza de Oro. Entrevista de Natasa Govekar con Marko Ivan Rupnik sobre arte, fe y evangelización. Burgos: Monte Carmelo.

Guardini, R. (1960). Imagen de culto e imagen de devoción. La esencia de la obra de arte. Madrid: Ediciones Guadarrama.

Ivanov, V. I. (1947). «I limiti dell'arte», en: Lo Gatto, E. (ed.), L'estetica e la poetica in Russia. Firenze: G. C. Sansoni-Editore, pp. 450-470

Ivanov, V. I. (1947). "Il simbolismo e la grande arte», en: Lo Gatto, E. (ed.), L'estetica e la poetica in Russia. Firenze: G. C. Sansoni-Editore, pp. 478-481.

Ivanov, V. (1987). Il grande libro delle icone russe. Milano: Edizione Paoline,.

Lipovetsky, G. y Serroy, J. (2015). La estetización del mundo. Vivir en la época del capitalismo artístico. Barcelona: Anagrama.

López Sáez, F. J. (2008). La belleza, memoria de la resurrección. Teodicea y antropodicea en Pavel Florenskij, Burgos: Monte Carmelo.

Mattei, G. (2009). Il cardinale Tomás Špidlik si racconta alla vigilia del suo compleanno. Da novant'anni con lo sguardo verso oriente, "L'Osservatore Romano». Recuperado de http://www.vatican.va/news_services/or/or_quo/interviste/2009/290q08a1.html [consultado el 2.03.2018]

Mattei, P. (2008). «Un arte que despierta veneración», 30DIAS. Recuperado de http:// www.30giorni.it/articoli_id_17853_12.htm [consultado el 1.07.2018].

Muzj, M. G. (2013). "Vita nello spirito e simbolo: una relazione necessaria nel pensiero di Ch. A. Bernard», en: Muzj, M.G. (ed.), Simbolo cristiano e linguaggio umano. Per una piena reintegrazione della teologia simbolica nella teologia. Milano: Vita e pensiero, pp. 147-167.

Plazaola, J. (1987). El arte y el hombre de hoy. Valladolid: Servicio de publicaciones de la Diputación Provincial de Valladolid.

Plazaola, J. (2006). Arte sacro actual. Madrid: BAC.

Ratzinger, J. (2006). La belleza. La Iglesia. Madrid: Ediciones Encuentro.

Rodríguez Velasco, M. (ed.) (2013). Tradición y Modernidad en la obra de Marko Iván Rupnik. Madrid: CEU Ediciones. 
Rupnik, M. I. (1987). Vassilij Kandinkij come approccio a una lettura del significato teologico dell'arte moderna alla luce della teología russa, Licenza in missiologia della Pontificia Università Gregoriana. Direttore: Heinrich Pfeiffer, Roma.

Rupnik, M. I. (1994). L'arte memoria della comunione. Il significato teologico missionario dell'arte nella saggistica di Vjačeslav Ivanovič Ivanov. Roma: Lipa.

Rupnik, M. I. (1995). «Per una teologia dell'arte ossia la teologia come creazione artistica», en: Špidlík, T. y Rupnik, M. I. (ed.), Parola e immagine. Roma: Lipa, pp. 68-125.

Rupnik, M. I. (2000). «L'artista dell'arte per il culto» en: Arte Cristiana 797, pp. 107-109.

Rupnik, M. I. (2000). «L'icona, l'invisibile e la storia» en: Euntes Docete 2, pp. 89-97.

Rupnik, M. I. (2002). "Cómo me he acercado al mosaico de la Capilla», en: Apa, M., Clément, O. y Valenziano, V. (ed.), La Capilla Redemptoris Mater del Papa Juan Pablo II. Burgos: Monte Carmelo, pp. 179-184.

Rupnik, M. I. (2003). «A partir de la belleza», en: Špidlik, T. y Rupnik M. I. (ed.), Teología de la evangelización desde la belleza. Madrid: BAC, pp. 519-543.

Rupnik, M. I. (2004). «Il simbolo: la proposta dei cristiani per l'arte», en: Ricci, F. C. (ed), Il cristianesimo fonte perenne di ispirazione per le arti. Napoli: Edizioni Scientifiche italiane, pp. 153-159.

Rupnik, M. I. (2005). «Applicazione del Concilio: quale arte per la liturgia?» en: Notitiae, 471-472, pp. 579-587.

Rupnik, M. I. (2006). «Verso un'arte sapienziale», en: Guzzi, M. (ed.), Lo spartiacque. Ciò che nasce e ciò che muore a Occidente. Milano: Paoline, pp. 161-176

Rupnik, M. I. (2009). «Implicaciones teológicas del mosaico», en: Toraño, E. y Prades, J. (ed.), Dios es amor. Madrid: San Dámaso, pp. 61-71.

Rupnik, M. I. (2010). "Bellezza e pensiero teologico», en: Ravasi, G. y Rupnik, M. I. (ed.), Il fascino del bello. Tra Bibbia e teologia. Milano: San Paolo, pp. 26-80.

Rupnik, M. I.(2010). «L'arte formatrice del cuore sacerdotale» en: Seminarium, A. L., 1, pp. 159-178.

Rupnik, M. I. (2011). «L’arte sacra e la liturgia», en: Verdon, T. (ed.), Bellezza e vita. La spiritualità nell'arte contemporanea. Milano: San Paolo, pp. 117-148.

Rupnik, M. I. (2011). «Presentazione», en: Farchione, A., Arte e Chiesa. La volontà di un incontro della Chiesa, l'attrazione per lo scontro dell'arte contemporanea. Verona: Fede\&Cultura, pp. 5-11.

Rupnik, M. I. (2013). Lectio Academica. La belleza, lugar del conocimiento integral, en: Rupnik, M. I. (ed.), Una belleza para el encuentro. Madrid: Universidad Francisco de Vitoria, pp. 43-52.

Rupnik, M. I. (2013). Una belleza para el encuentro. Madrid: Universidad Francisco de Vitoria.

Rupnik, M. I. (2015). L'autoritratto della Chiesa. Arte, bellezza e spiritualità. Roma: EDB.

Rupnik, M. I. (2015). «Le chiese, icone della Chiesa» en: Thema. Rivista dei Beni Culturali Ecclesiastici 5, pp. 16-20.

Ruiz de Loizaga Martín, M. (2017). «Hacia un arte litúrgico, según la concepción de Marko Ivan Rupnik. Reflexiones sobre el arte sacro» en: Scripta Theologica 49, pp. 619-643.

Ruiz de Loizaga Martín, M. (2017). «La semplificazione delle forme. Influenza di Matisse nei mosaici di Marko Ivan Rupnik e il Centro Ezio Aletti» en Collectanea Franciscana, 87, pp. 613-634.

Ruiz de Loizaga Martín, M. (2019). «La reinterpretación del descenso de Cristo a los infiernos en los mosaicos litúrgicos del Centro Aletti» en Estudios Eclesiásticos, 94, pp. 443-475. 
Schmemann, A. (2014). Chiesa, mondo e missione. Roma: Lipa.

Solov'ëv, V. (1988). «Il significato universale dell'arte», en: Dell'Asta, A. (ed.), Il significato dell'amore e altri scritti. Milano: Edizioni R. C. La Casa di Matriona, pp. 149-162.

Solov'ëv, V. (1998). I fondamenti spirituali della vita. Roma: Lipa.

Sotoo, E. y Almuzara, J. M. (2010). De la piedra al Maestro. Madrid: Palabra.

Špidlík, T. (1986). Los grandes místicos rusos. Madrid: Ciudad Nueva.

Špidlík, T. (2006). Alle fonti dell'Europa. In principio era l'arte. Roma: Lipa.

Špidlík, T. (2008). Presentación, en: López Sáez, F. J. (ed.), La belleza, memoria de la resurrección. Teodicea y antropodicea en Pavel Florenskij. Burgos: Monte Carmelo, pp. 9-12.

Špidlík, T. y Rupnik, M. I. (2003). La fe según los iconos. Burgos: Monte Carmelo.

Špidlík, T. y Rupnik, M. I. (2013). El conocimiento integral. La vía del símbolo. Madrid: BAC.

Universidad Pontificia Comillas (28.10.2015), Lección Magistral de Teología. Marko Rupnik. Discernimiento de la cultura a través del arte [Archivo de video]. Recuperado de: https://tv.comillas.edu/media/Lecci\%C3\%B3n+Magistral+de+Teolog\%C 3\%ADa\%2C+Marko+Rupnik.+ +3+11+2015/1_mxf6bwh0/66601841 [Consultado el 3.12.2016]

Velasco Quintana, P. y Rodríguez Velasco, M. (2009). «Marko Ivan Rupnik. Una nueva forma de creación frente al subjetivismo contemporáneo» en: Debate Actual 13, pp. 118-127.

Von Balthasar, H. U. (1992). «Arte cristiano y predicación», en: Feiner, J. y Löhrer, M. (ed.), Mysterium Salutis. Manual de Teología como historia de la salvación, I. Madrid: Ediciones Cristiandad, pp. 774-792.

Universidad San Pablo-CEU (Madrid)

María Ruiz de Loizaga Martín

Facultad de Humanidades y CC. de la Comunicación

maria.ruizloizagamartin@ceu.es

ID ORCID: 0000-0002-9264-5011

[Artículo aprobado para publicación en enero de 2019] 